$2^{\text {nd }}$ International Workshop on Advanced Smart Materials and Smart Structures Technology

Gyeong-ju, Korea, July 21-24, 2005

\title{
New Results in the Use of Piezoelectric Wafer Active Sensors for Structural Health Monitoring
}

\author{
Victor Giurgiutiu \\ ${ }^{1}$ University of South Carolina, Columbia, SC 29208, victorg@sc.edu
}

Piezoelectric-wafer active sensors (PWAS) are small, inexpensive, non-invasive, elastic wave generators/receptors that can be easily affixed to a structure. Piezoelectric-wafer active sensors are wideband non-resonant devices. They can be wired into sensor arrays and connected to data concentrators and wireless communicators. However, the development of PWAS technology is not yet complete, and a number of issues have still to be resolved. This paper presents new results obtained in using the PWAS for structural health monitoring. The new results to be presented were obtained in the following directions: (i) tuning the PWAS of asymmetric geometries (rectangular plan forms) to excite and detect different Lamb wave modes in selective directions; (ii) PWAS durability and survivability; (iii) steering beam large-area coverage (embedded ultrasonic structural radar - EUSR); and probability of detection (POD) with PWAS probes (an initial investigation). The paper presents analytical methods and experimental results that can be successfully used in the design of active SHM systems to be installed on operational structures. The durability and survivability of the PWAS transducers has been tested under various environmental exposures and the importance of adequate adhesive and protective coating design has been shown. These initial results are sufficient to gain confidence in this new technology and start installation on realistic structures. (For longer-term durability and survivability results, further tests are planned in subsequent studies.) The EUSR steering beam method based on PWAS phased-arrays has been refined to increase it power of detection for various structural defects. It has been also proved that EUSR performance is not affected by structural curvature typical of aircraft installation. The initial investigation of PWAS POD has indicated that crack as small as $1.57 \mathrm{~mm}$ can be detected directly and imaged relatively easily. Overall, these results confirm that PWAS transducers are a technology with good potential for implementation in active SHM systems. The paper will finish with a summary of the presented work and a perspective for further research and industrial implementation.

\section{INTRODUCTION}

Embedded nondestructive evaluation (NDE) is an emerging technology that will allow the transition of the conventional ultrasonics methods to embedded structural health monitoring (SHM) systems such as those envisioned for vehicle health management (VHM). SHM for VHM requires the development of small, lightweight, inexpensive, unobtrusive, minimally invasive sensors to be embedded in the airframe with minimum weight penalty and at affordable costs. Such sensors should be able to scan the structure and identify the presence of defects and incipient damage. Current ultrasonic inspection of thin wall structures (e.g., aircraft shells, storage tanks, large pipes, etc.) is a time consuming operation that requires meticulous through-the-thickness C-scans over large areas. One method to increase the efficiency of thinwall structures inspection is to utilize guided waves (e.g., Lamb waves) instead of the conventional

\footnotetext{
${ }^{1}$ Professor
} 
pressure waves. Guided waves propagate along the mid-surface of thin-wall plates and shallow shells. They can travel at relatively large distances with very little amplitude loss and offer the advantage of large-area coverage with a minimum of installed sensors. Guided Lamb waves have opened new opportunities for cost-effective detection of damage in aircraft structures, and a large number of papers have recently been published on this subject. Traditionally, guided waves have been generated by impinging the plate obliquely with a tone-burst from a relatively large ultrasonic transducer. Snell's law ensures mode conversion at the interface, hence a combination of pressure and shear waves are simultaneously generated into the thin plate. However, conventional Lamb-wave probes (wedge and comb transducers) are relatively too heavy and expensive to consider for widespread deployment on an aircraft structure as part of a SHM system. Hence, a different type of sensors than the conventional ultrasonic transducers is required for the SHM systems. Piezoelectric wafer active sensors (PWAS) are inexpensive, non-intrusive, un-obtrusive devices that can be used in both active and passive modes. In active mode, PWAS generated Lamb waves that can be used for damage detection through pulse-echo or pitch-catch techniques.

\section{GUIDED-WAVE GENERATION/DETECTION WITH PIEZOELECTRIC WAFER ACTIVE SENSORS}

In conventional ultrasonics, guided waves are generated by impinging the structural surface obliquely with a ultrasonic beam from a relatively large ultrasonic transducer affixed to a wedge. Snell's law ensures mode conversion at the interface, hence a combination of pressure and shear waves are simultaneously generated into the structure. If the structure is thin walled, guided plate or shell waves are being created. Alternatively, guided waves can be generated with a comb transducer, having comb spacing tuned with the guided-wave half wavelength. However, these conventional Lamb-wave probes (wedge and comb transducers) are relatively heavy, bulky, and expensive. In recent years, piezoelectric wafers permanently attached to the structure have been used for the guided waves generation and detection. For lack of a better term, we have named these devices piezoelectric wafer active sensors (PWAS). PWAS operated on the piezoelectric principle that couples the electrical and mechanical variables in the material (mechanical strain, $S_{i j}$, mechanical stress, $T_{k l}$, electrical field, $E_{k}$, and electrical displacement $D_{j}$ ) in the form:

$$
\begin{aligned}
& S_{i j}=s_{i j k l}^{E} T_{k l}+d_{k i j} E_{k} \\
& D_{j}=d_{j k l} T_{k l}+\varepsilon_{j k}^{T} E_{k}
\end{aligned}
$$

where $s_{i j k l}^{E}$ is the mechanical compliance of the material measured at zero electric field $(E=0), \varepsilon_{j k}^{T}$ is the dielectric permittivity measured at zero mechanical stress $(T=0)$, and $d_{k i j}$ represents the piezoelectric coupling effect. For embedded NDE applications, PWAS couple their in-plane motion, excited by the applied oscillatory voltage through the piezoelectric effect, with the Lamb-waves particle motion on the material surface. Lamb waves can be either quasi-axial (S0, S1, S2, ..), or quasi-flexural (A0, A1, A2, ...). Figure 1 shows the interaction between surface mounted PWAS and $S_{0}$ and $A_{0}$ guided Lamb waves.

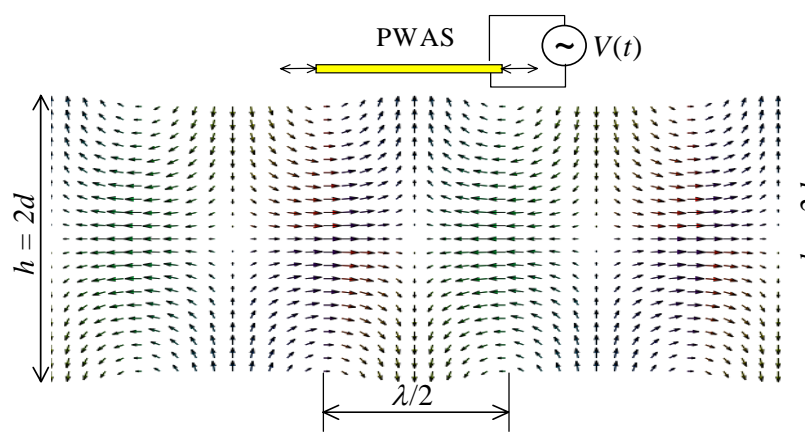

(a)

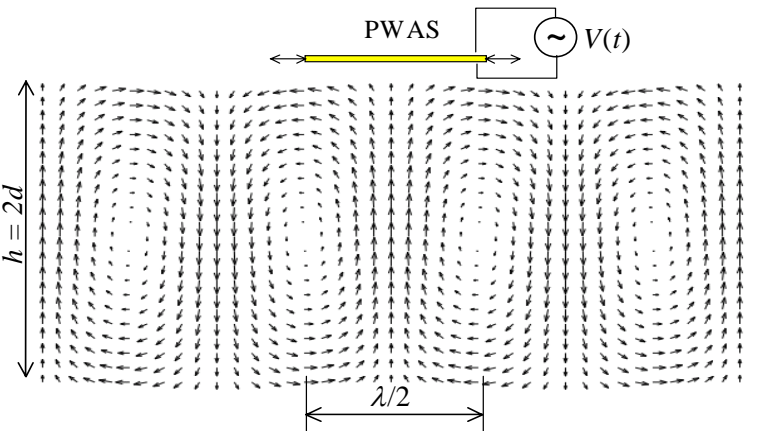

(b)

Figure 1 PWAS interaction with Lamb waves in a plate: (a) $S_{0}$ mode; (b) Ao mode 
The in-plane interaction between the PWAS and the guided Lamb waves is such that preferential tuning can be achieved when the representative PWAS dimensions are near an odd multiple of the half wavelength of certain Lamb-wave mode. Thus, multiple tuning can be achieved. Giurgiutiu (2003) developed the theory of the interaction of a rectangular PWAS with straight-crested Lamb waves and presented tuning prediction formulae based on trigonometric functions.

$$
\left.\varepsilon_{x}(x, t)\right|_{y=d}=-i \frac{a \tau_{0}}{\mu}\left[\sum_{\xi^{S}} \sin \left(\xi^{S} a\right) \frac{N_{S}\left(\xi^{S}\right)}{D_{S}^{\prime}\left(\xi^{S}\right)} e^{i\left(\xi^{S} x-\omega t\right)}+\sum_{\xi^{A}} \sin \left(\xi^{A} a\right) \frac{N_{A}\left(\xi^{A}\right)}{D_{A}{ }^{\prime}\left(\xi^{A}\right)} e^{i\left(\xi^{A} x-\omega t\right)}\right]
$$

where $\quad N_{S}=\xi \beta\left(\xi^{2}+\beta^{2}\right) \cos (\alpha d) \cos (\beta d) ; N_{A}=\xi \beta\left(\xi^{2}+\beta^{2}\right) \sin (\alpha d) \sin (\beta d)$

$$
\begin{aligned}
& D_{S}=\left(\xi^{2}-\beta^{2}\right)^{2} \cos (\alpha d) \sin (\beta d)+4 \xi^{2} \alpha \beta \sin (\alpha d) \cos (\beta d) \\
& D_{A}=\left(\xi^{2}-\beta^{2}\right)^{2} \sin (\alpha d) \cos (\beta d)+4 \xi^{2} \alpha \beta \cos (\alpha d) \sin (\beta d)
\end{aligned}
$$

$\xi^{S}$ and $\xi^{A}$ are the zeros of $D_{S}$ and $D_{A}$ respectively. We can note that these are the solutions of the Rayleigh-Lamb equation.

Raghavan and Cesnik (2004) extended these results to the case of a circular transducer coupled with circular-crested Lamb waves and proposed corresponding tuning prediction formulae based on Bessel functions:

$$
\left.\varepsilon_{r}(r, t)\right|_{z=d}=\pi \frac{\tau_{0} a}{\mu} e^{i \omega t}\left[\sum_{\xi^{S}} J_{1}\left(\xi^{S} a\right) \xi^{S} \frac{N_{S}\left(\xi^{S}\right)}{D_{S}{ }^{\prime}\left(\xi^{S}\right)} H_{1}^{(2)}\left(\xi^{S} r\right)+\sum_{\xi_{A}} J_{1}\left(\xi^{A} a\right) \xi^{A} \frac{N_{A}\left(\xi^{A}\right)}{D_{A}{ }^{\prime}\left(\xi^{A}\right)} H_{1}^{(2)}\left(\xi^{A} r\right)\right]
$$

A comprehensive study of these prediction formulae in comparison experimental results has recently been performed by Bottai and Giurgiutiu (2005). Experiments were performed on large aluminum alloy plates using square, circular, and rectangular PWAS at frequencies up to $700 \mathrm{kHz}$. Two plate thickness were studied, $1.07 \mathrm{~mm}$ and $3.15 \mathrm{~mm}$. In the thinner plate, only two Lamb wave modes, $\mathrm{A}_{0}$ and $\mathrm{S}_{0}$ were present in the explored frequency range. In the thicker plate, a third Lamb wave, $\mathrm{A}_{1}$, was also present.

Thin-plate Experiments with Square and Circular PWAS

Figure 2 shows the results for a 7-mm square PWAS placed on 1.07-mm 2024-T3 aluminum alloy plate. The experimental results (Figure $2 \mathrm{a}$ ) show that a rejection of the highly dispersive $\mathrm{A}_{0}$ Lamb wave mode is observed at around $200 \mathrm{kHz}$. At this frequency, only the $\mathrm{S}_{0}$ mode is excited, which is very beneficial for pulse-echo studies due to the low dispersion of the $S_{0}$ mode at this relatively low value of the $f d$ product. On the other hand, a strong excitation of the $\mathrm{A}_{0}$ mode is observed at around $50 \mathrm{kHz}$. These experimental results were reproduced using Equation (2) with the assumption that the effective PWAS length is $6.4 \mathrm{~mm}$ (Figure 2b). The difference between the actual PWAS length and effective PWAS length is attributed to shear transfer/diffusion effects at the PWAS boundary.

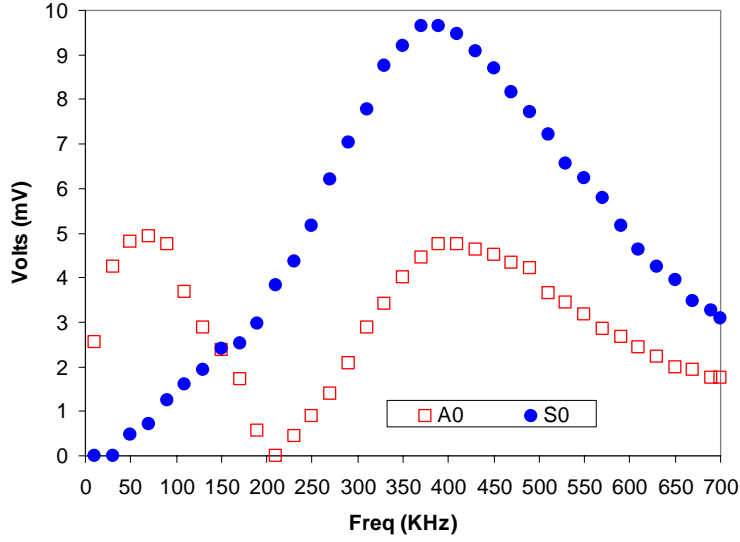

(a)

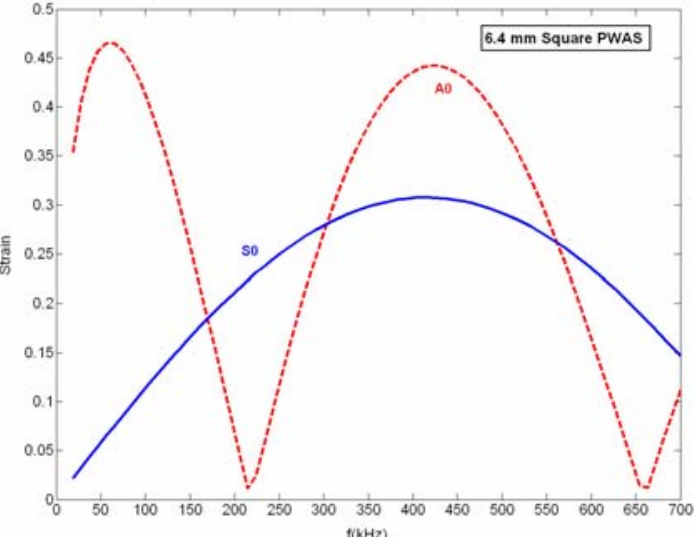

(b)

Figure 2: Lamb-wave tuning using a 7-mm square PWAS placed on 1.07-mm 2024-T3 aluminum alloy plate: (a) experimental results; (b) prediction with Equation (2) for $6.4 \mathrm{~mm}$ effective PWAS length. 
The results for the circular PWAS showed a rejection of the highly dispersive $\mathrm{A}_{0}$ Lamb wave mode at around $300 \mathrm{kHz}$. At this frequency, only the $S_{0}$ mode is excited, which is very beneficial for pulse-echo studies due to the low dispersion of the $\mathrm{S}_{0}$ mode at this relatively low value of the $f d$ product. On the other hand, a strong excitation of the $A_{0}$ mode is observed at around $50 \mathrm{kHz}$. These experimental results were reproduced using Equation (3) with the assumption that the effective PWAS length is $6.4 \mathrm{~mm}$ (Figure 2b). The difference between the actual PWAS length and effective PWAS length is attributed to shear transfer/diffusion effects at the PWAS boundary.

It worth noticing that for the 7-mm square PWAS the $S_{0}$ "sweet spot" happens at approximately 200 $\mathrm{kHz}$, whereas for the 7-mm round PWAS the $\mathrm{S}_{0}$ "sweet spot" happens at approximately $300 \mathrm{kHz}$. On the other hand, the $\mathrm{A}_{0}$ sweet spot happened in both cases at the same frequency of approximately $50 \mathrm{kHz}$. The difference between these two frequency can be attributed to a number of concurrent factors such as: (a) the different assumption that underline Equations (2) and (3); (b) the corner effects in the case of the rectangular PWAS. It is also remarkable that, in spite of these differences, the equivalent PWAS length is the same $(6.4 \mathrm{~mm})$ in both cases.

\section{Thick-plate Experiments with Square and Circular PWAS}

Figure 3 shows the results for a 7-mm square PWAS placed on 3.15-mm 2024-T3 aluminum alloy plate. The experimental results (Figure 3a) show that a partial rejection of the $\mathrm{A}_{0}$ Lamb wave mode is observed at around $350 \mathrm{kHz}$. At this frequency, the $\mathrm{S}_{0}$ mode is predominantly excited. However, do the higher values of the $f d$ product, the $S_{0}$ mode is already dispersive at this frequency. In addition, it is noticed that the $\mathrm{A}_{0}$ mode is only partially rejected at this frequency, and hence it is still present in the signal. For these reasons, this $\mathrm{S}_{0}$ sweet spot is not as effective in thick plates as it were in the thin plates described in the previous section. On the other hand, a strong excitation of the $\mathrm{A}_{0}$ mode is observed at around $100 \mathrm{kHz}$. Again, this is accompanied by the partial excitation of the other mode, the $S_{0}$ mode. In addition, a third Lamb-wave mode becomes excited at higher frequencies, i.e., above $500 \mathrm{kHz}$. These three observations indicate that the excitation of Lamb waves with PWAS transducers is much more complex in thick plates than in thin plates. These experimental results were reproduced using Equation (2) with 7-mm effective PWAS length (Figure 3b).

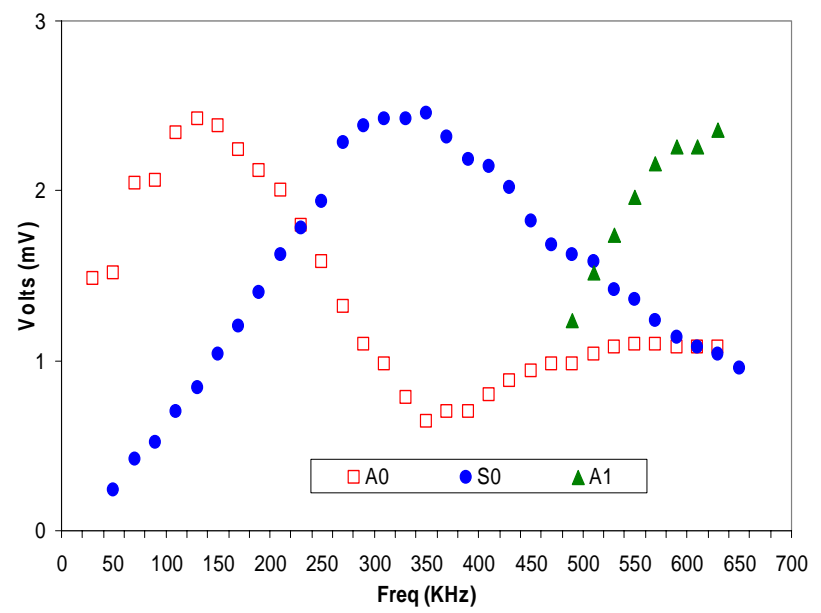

(a)

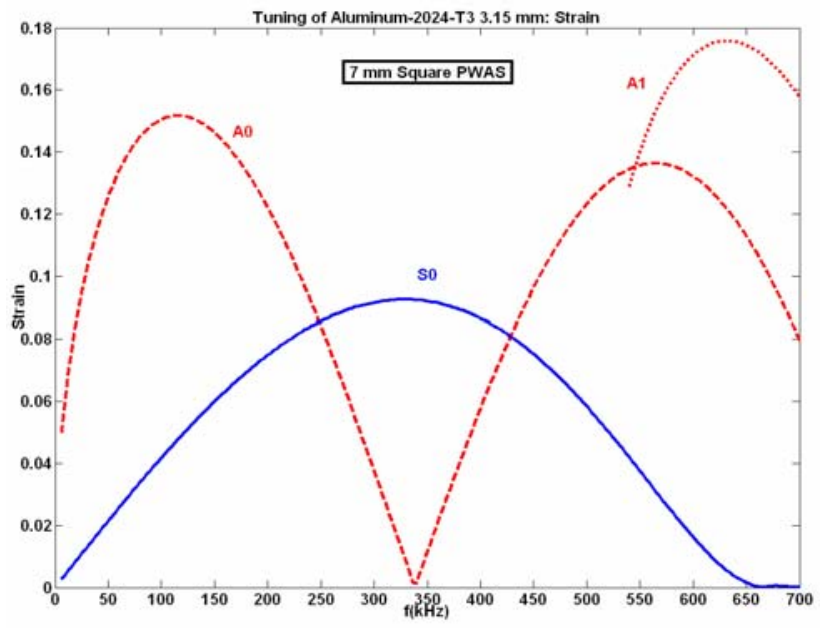

(b)

Figure 3: Lamb-wave tuning using a 7-mm square PWAS placed on 3.15-mm 2024-T3 aluminum alloy plate: (a) experimental results; (b) prediction with Equation (2) for $6.4 \mathrm{~mm}$ effective PWAS length.

The results for the circular PWAS are shown in Figure 4. The experimental results (Figure 4a) show that a partial rejection of the $A_{0}$ Lamb wave mode is observed at around $420 \mathrm{kHz}$. At this frequency, the $\mathrm{S}_{0}$ mode is more strongly excited than the $\mathrm{A}_{0}$ mode. However, do the higher values of the $f d$ product, the $\mathrm{S}_{0}$ mode is already dispersive at this frequency. In addition, it is noticed that the $\mathrm{A}_{0}$ mode, which is only partially, is still present in the signal. For these reasons, this $S_{0}$ sweet spot is not as effective in thick plates as it were in the thin plates described in the previous section. On the other hand, a strong excitation 
of the $\mathrm{A}_{0}$ mode is observed at around $150 \mathrm{kHz}$. Again, this is accompanied by the partial excitation of the other mode, the $\mathrm{S}_{0}$ mode. In addition, a third Lamb-wave mode becomes excited at higher frequencies, i.e., above $450 \mathrm{kHz}$. These three observations indicate that the excitation of Lamb waves with PWAS transducers is much more complex in thick plates than in thin plates. These experimental results were reproduced using Equation (3) with 7-mm effective PWAS length (Figure 3b).

It worth noticing that in the case of a thick plate the partial rejection of the $\mathrm{A}_{0}$ mode happened at 350 $\mathrm{kHz}$ for the 7-mm square PWAS and at $420 \mathrm{kHz}$ for the 7-mm round PWAS. These frequencies are much closer to each other than in the case of the thin plate. On the other hand, the $\mathrm{A}_{0}$ sweet spot happened at $100 \mathrm{kHz}$ for the square PWAS and at $150 \mathrm{kHz}$ for the round PWAS. This is very different from the thin plate, where the $A_{0}$ mode sweet spot happened at about the same frequency of $50 \mathrm{kHz}$. These observations indicate that simple scale rules do not apply due to the highly nonlinear character of the characteristic equations governing the Lamb waves and a full analysis is required in order for accurate predictions to be achieved.

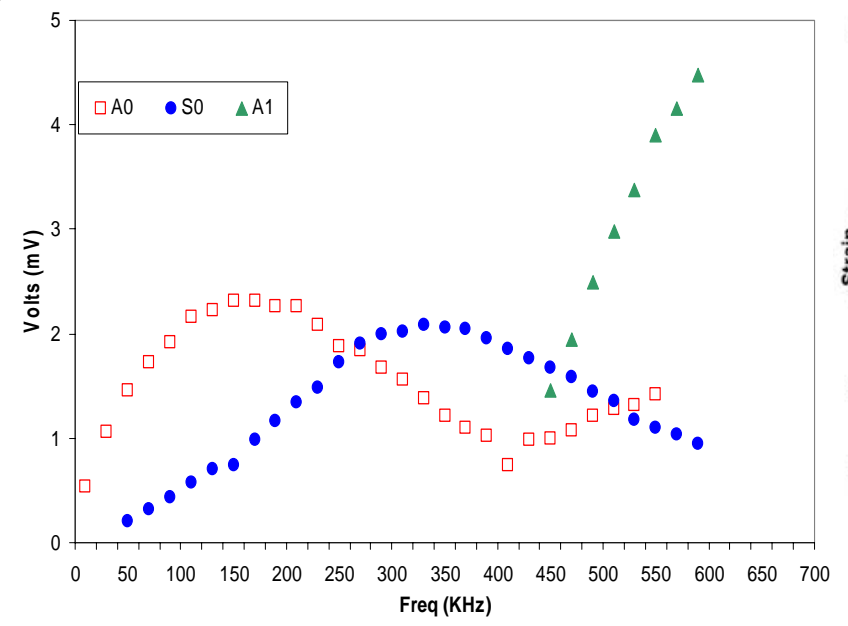

(a)

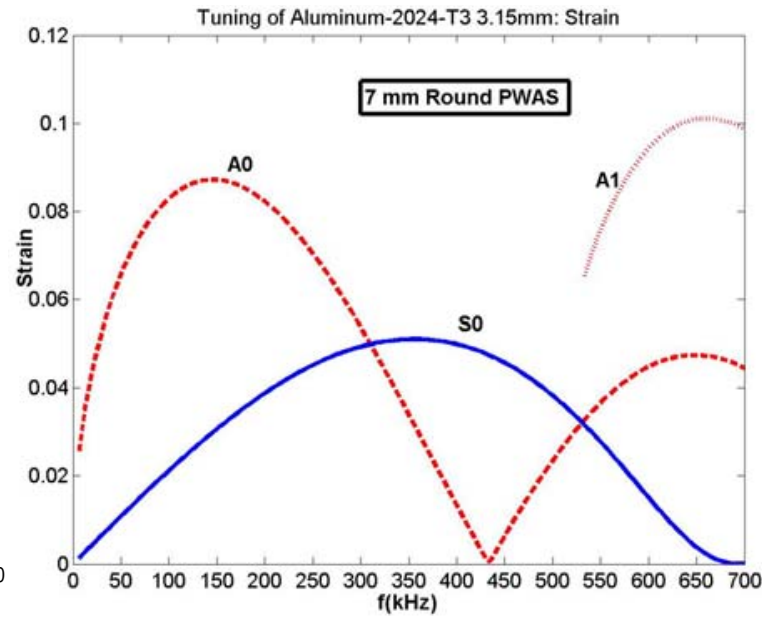

(b)

Figure 4: Lamb-wave tuning using a 7-mm round PWAS placed on 3.15-mm 2024-T3 aluminum alloy plate: (a) experimental results; (b) prediction with Equation (3) for 7-mm effective PWAS length.

\section{Experiments with Rectangular PWAS}

Rectangular PWAS of high aspect ratio (5:1) were tested to explore the directional tuning of Lamb waves with PWAS transducer. Three rectangular PWAS of $25 \mathrm{~mm} \times 5 \mathrm{~mm}$ size, and $0.15 \mathrm{~mm}$ thickness (Steiner \& Martin) were placed in an L-configuration. PWAS P1 (a the center of the L) was the transmitter and PWAS P2 and P3 (end of the L legs) were the receivers. Two sets of experiments were performed: (i) P1 transmitting to P2; (ii) P1 transmitting to P3. In the first case, lengthwise tuning was explored. In the second case, widthwise tuning was explored. Since the difference between length and width are very significant (5:1 ratio) considerable different tuning behavior was expected.

Figure 5 shows the result for lengthwise tuning. Since the PWAS is relatively long, the tuning frequencies are relatively low. Hence, the frequency range was restricted to $250 \mathrm{kHz}$ and the frequency step was reduced such that a finer exploration can be performed. Figure 5a presents the experimental results. Three valleys are observed in the $\mathrm{A}_{0}$ mode at approximately $85 \mathrm{kHz}, 135 \mathrm{kHz}$, and $175 \mathrm{kHz}$. A peak in the $\mathrm{A}_{0}$ mode is observed at around $45 \mathrm{kHz}$. On the other hand, the $\mathrm{S}_{0}$ mode seems to peak at around $110 \mathrm{kHz}$. This behavior could be reproduced relatively well using Equation (2) with an effective PWAS length of $24.8 \mathrm{~mm}$. (Figure 5b). The only point that could not be reproduced acceptably seems to be the third $\mathrm{A}_{0}$ valley.

Figure 6 shows the results for widthwise tuning. Since the width is relatively small, the tuning frequencies are expected to be relatively high. Figure $6 a$ indicates that one $A_{0}$ rejection frequency is observed at around $530 \mathrm{kHz}$. At this frequency, the $\mathrm{S}_{0}$ mode, which is almost non dispersive at this value of the $f d$ product, is dominant. The $\mathrm{A}_{0}$ mode, on the other hand, is found to be dominant at around 100 
kHz. This behavior was reproduced quite well with Equation (2) using an effective PWAS length of 4.5 $\mathrm{mm}$.

These studies have proved that high-aspect ratio rectangular PWAS is able to preferentially tune certain Lamb wave modes in one direction and other Lamb wave modes in the other direction.

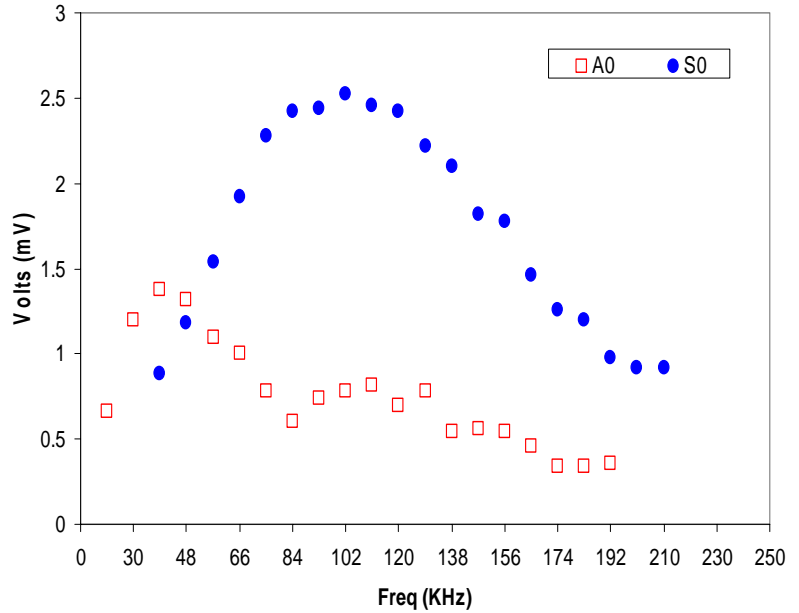

(a)

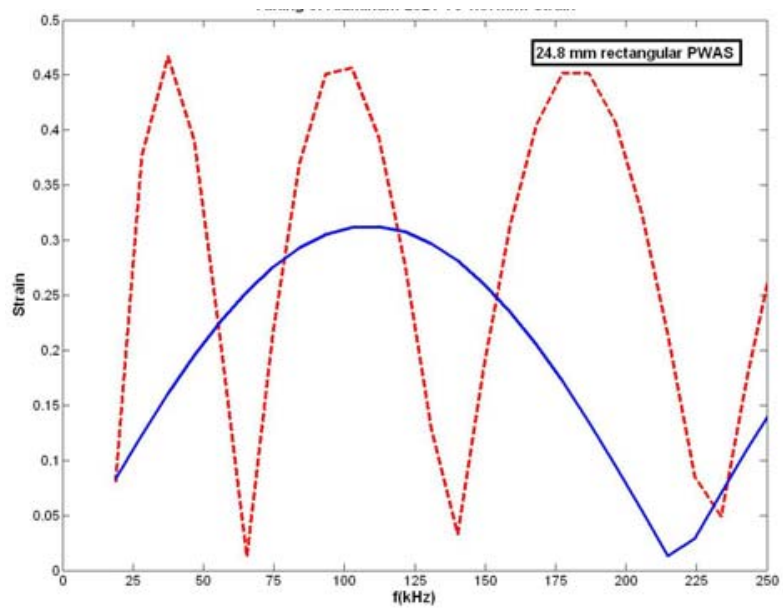

(b)

Figure 5: Lengthwise Lamb-wave tuning with a 25-mm by 5-mm rectangular PWAS placed on a 1.07-mm 2024-T3 aluminum alloy plate: (a) experimental results; (b) prediction with Equation (2) for 24.8-mm effective length.

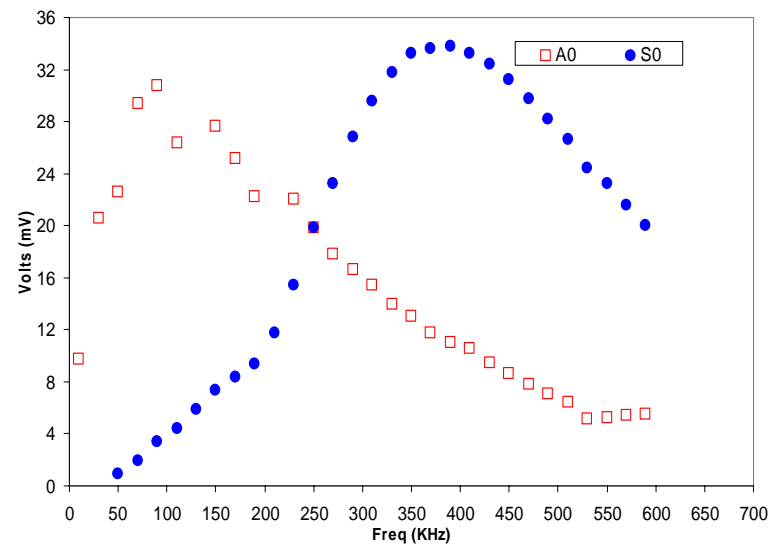

(a)

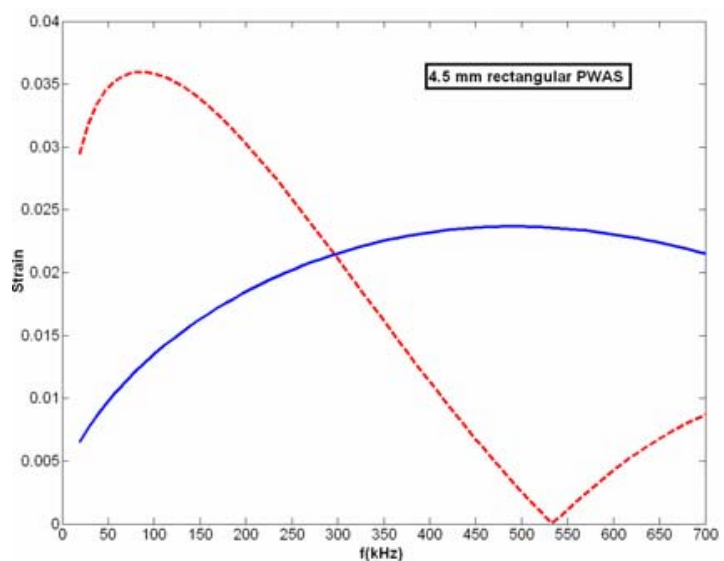

(b)

Figure 6: Widthwise Lamb-wave tuning with a $25-\mathrm{mm}$ by 5-mm rectangular PWAS placed on a 1.07-mm 2024-T3 aluminum alloy plate: (a) experimental results; (b) prediction with Equation (2) for 4.5-mm effective length.

\section{DURABILITY AND SURVIVABILITY OF PIEZOELECTRIC WAFER ACTIVE SENSORS}

A number of studies have been performed to assess PWAS durability and survivability under exposure to environmental factors, large strains, and fatigue cyclic loads. Both free PWAS and PWAS attached to an aluminum alloy plate were used. The PWAS behavior was monitored using the of the electromechanical (E/M) impedance method. For a free PWAS, the real part of the E/M impedance reflects its free vibration spectrum. For a bonded PWAS, The real part of the $\mathrm{E} / \mathrm{M}$ impedance directly reflects the vibration spectrum of the tested specimen seen through the point-wise mechanical impedance at the point where PWAS is attached to the structure (Giurgiutiu and Zagrai, 2002).

Doane and Giurgiutiu (2005) studied the behavior of PWAS transducers under large strains and under fatigue cyclic loading. To test the characteristics of the PWAS under large strain conditions, a PWAS was bonded to aircraft grade 2024-T3 aluminum alloy test specimen and subjected to tensile loading. In the large strain experiments, the specimen shown in Figure 7a was used (Doane and Giurgiutiu, 2005). The 
specimens were fabricated from $2024 \mathrm{~T} 3$ aluminum with a nominal thickness of $1 \mathrm{~mm}$. The specimens were loaded in tension under strain control. Two specimens were used: the first one was loaded up to 5000 micro-strain, the other up to failure. Measurements were taken at 200 micro-strain intervals. The baseline impedance was recorded at zero strain and additional readings were recorded until failure of the PWAS occurred. Minimal changes occurred to the impedance signature until the value of 3000 microstrain was exceeded. Significant changes begin to happen after 3000 micro-strain. After 6000 microstrain, the changes in the E/M impedance were very strong. Eventually the PWAS failed in tension at approximately 7200 micro-strain (Doane and Giurgiutiu, 2005). The PWAS failure was in the form of a transverse crack.

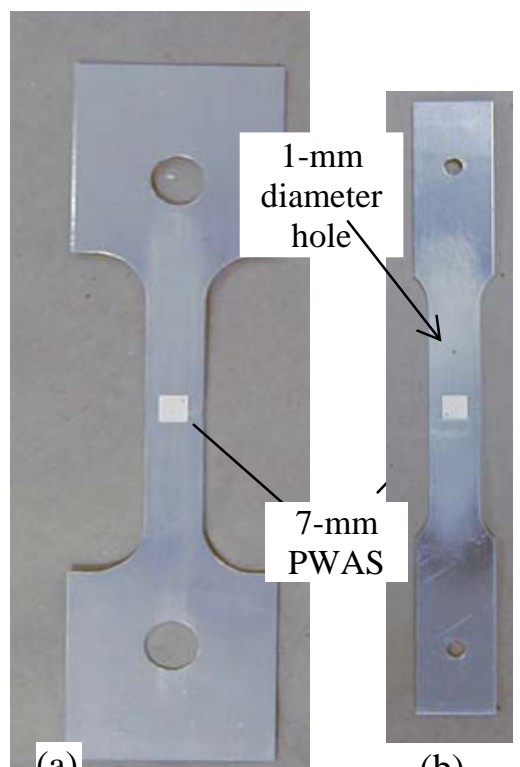

(a) (b)

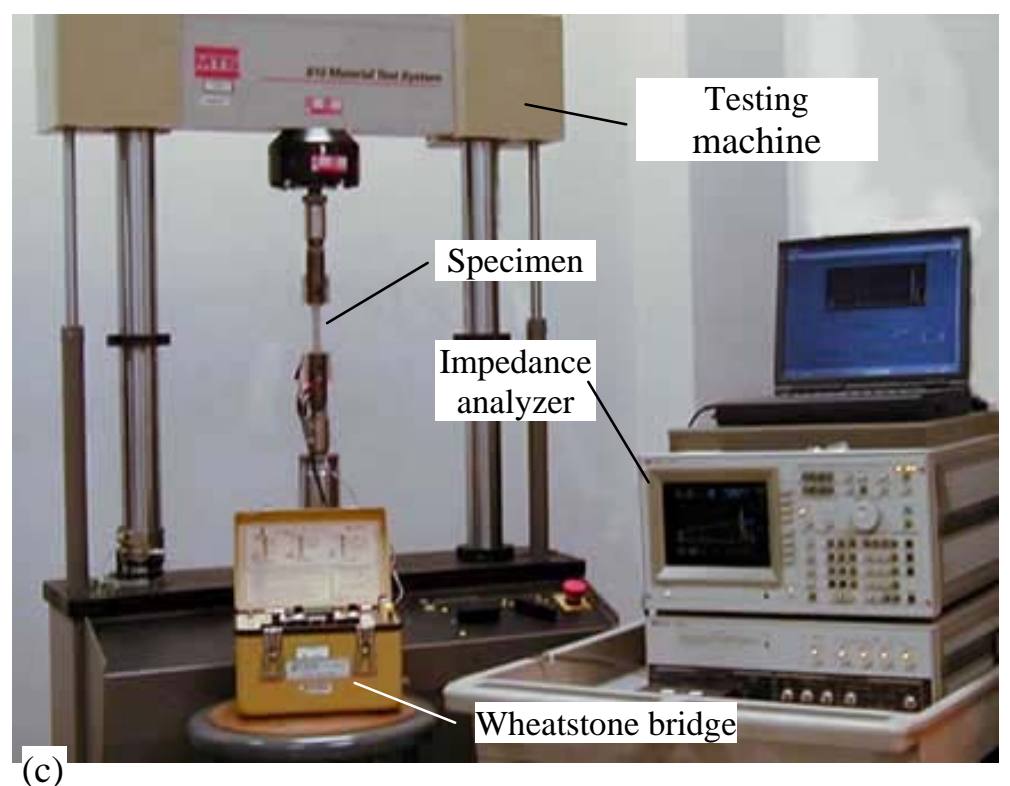

(c)

Figure 7 Large strains and fatigue cyclic loading tests: (a) large-strain test specimen; (b) fatigue cycling loading test specimen; (c) experimental setup (Doane and Giurgiutiu, 2005)

In the fatigue cyclic loading experiments, the specimen shown in Figure $7 \mathrm{~b}$ was used (Doane and Giurgiutiu, 2005). The specimens were fabricated from 2024 T3 aluminum with a nominal thickness of 1 mm. A 7-mm square PWAS was bonded to the specimen with M-Bond 200 cyanoacrylate adhesive. A 1$\mathrm{mm}$ hole was drilled in the specimen to act as stress concentration and localize the fatigue failure. Five specimens were used (Table 1). The specimens were loaded in fatigue cyclic loading with the mean loads and amplitudes adjusted such as to cause failure of the aluminum substrate at various values between 100 thousand and 10 million cycles (Table 1). The baseline impedance reading was taken with the mean load applied at the beginning of the tests and at predetermined cyclic intervals. Small settle-in changes occurred in the impedance readings in the first 30 to 40 thousand cycles. Beyond this the PWAS readings were relatively unchanged until the metallic specimen finally broke under fatigue cyclic loading. The specimen failure always occurred at the 1-mm stress-concentration hole. The PWAS survived the fatigue failure of all the metallic specimens.

Table 1 Fatigue specimens overview $(\mathrm{R}=0.1)$ (Doane and Giurgiutiu, 2005)

\begin{tabular}{lrrrrr}
\hline \hline & PWAS F1 & PWAS F2 & PWAS F3 & PWAS F4 & PWAS F5 \\
\hline Max load & $2104 \mathrm{~N}$ & $1560 \mathrm{~N}$ & $1335 \mathrm{~N}$ & $1156 \mathrm{~N}$ & $1067 \mathrm{~N}$ \\
Min load & $210 \mathrm{~N}$ & $156 \mathrm{~N}$ & $134 \mathrm{~N}$ & $116 \mathrm{~N}$ & $107 \mathrm{~N}$ \\
Mean load & $1157 \mathrm{~N}$ & $858 \mathrm{~N}$ & $734 \mathrm{~N}$ & $636 \mathrm{~N}$ & $587 \mathrm{~N}$ \\
Cycles to specimen failure & $178 \mathrm{kc}$ & $670 \mathrm{kc}$ & $1.3 \mathrm{Mc}$ & $6.25 \mathrm{Mc}$ & $12.2 \mathrm{Mc}$ \\
\hline \hline
\end{tabular}



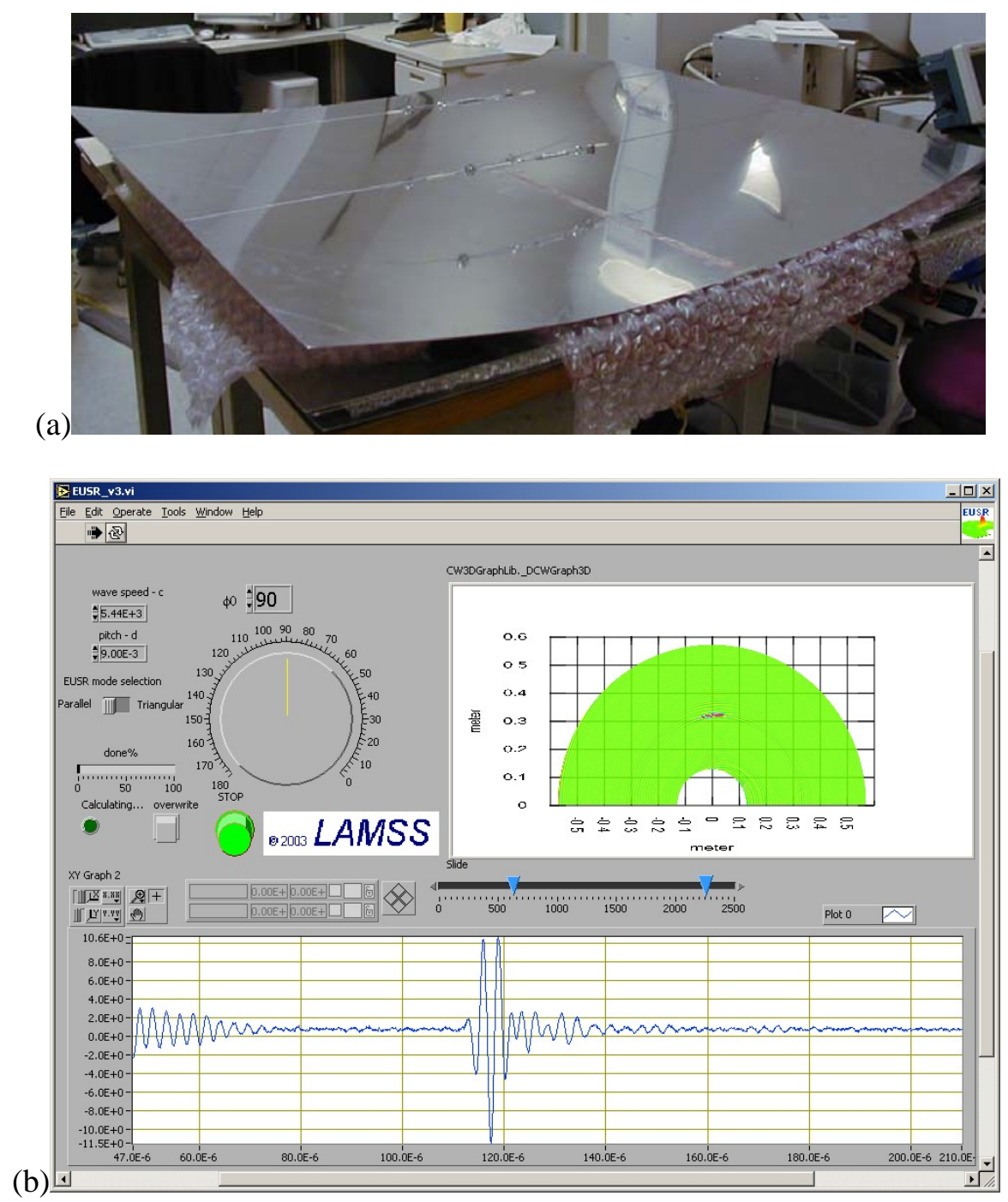

Figure 8 Crack detection at high curvature $(R=1.95 \mathrm{~m})$ in direction 1: (a) photo of specimen while being bend; (b) crack imaging and crack echo in the EUSR algorithm(Giurgiutiu, Yu, and Thomas, 2004)

\section{IMPROVED EUSR STEERING BEAM DETECTION}

The embedded ultrasonics structural radar (EUSR) is a phase-array application of the PWAS technology. The EUSR principles and initial results were reported extensively by Giurgiutiu and Bao (2002). In this section, we will report on recent work aimed at improving the EUSR steering beam detection of structural defects. Since the initial EUSR investigation was done on flat panels, and since most aircraft structures are curved, an initial concern was to verify that the EUSR algorithm works as well on curved thin-wall structures, such as shallow-curvature shells. Radii of curvature from $8.8 \mathrm{~m}$ down to $1.95 \mathrm{~m}$ were investigated Giurgiutiu, Yu, and Thomas, 2004). It was found that the curvature does not affect the EUSR power of detection. Figure 8 illustrates these results for the case of $\mathrm{R}=1.95 \mathrm{~m}$, which was the highest curvature tested. Thus, it was proved that EUSR performance is not affected by structural curvature typical of aircraft installation.

The EUSR steering beam method based on PWAS phased-arrays has been refined to increase it power of detection for various structural defects (Yu and Giurgiutiu, 2005). Simultaneous detection of two crack were placed symmetrically offside the PWAS phased array was achieved. Another concern was related to the probability of detection (POD) with the EUSR method. In an initial investigation, experiments were conducted to determine the smallest damage that the EUSR method could detect ( $\mathrm{Yu}$ and Giurgiutiu, 
2005). It was found that a pinhole as small as $1.57 \mathrm{~mm}$ can be detected directly and imaged relatively easily (Figure 9).

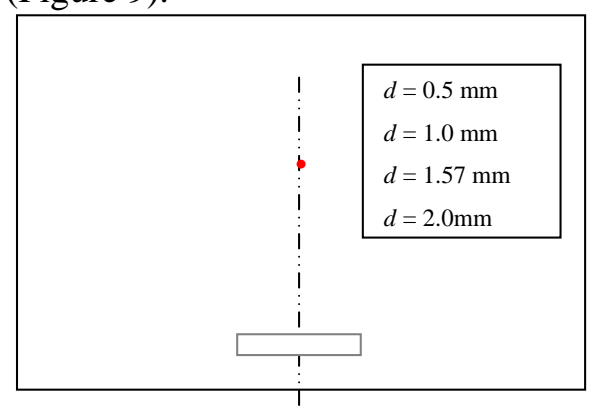

(a)

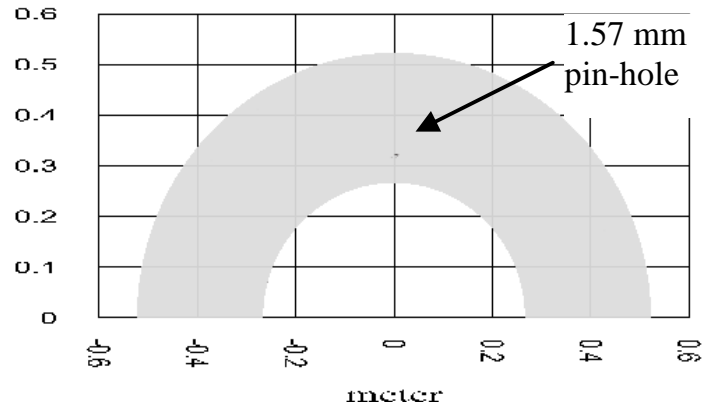

(b)

Figure 9 EUSR detection of pin-holes: (a) schematic of the experimental specimen; (b) EUSR GUI mapped image of the minimum detectable pin-hole with $1.57 \mathrm{~mm}$ diameter

\section{SUMMARY AND CONCLUSIONS}

This paper has discussed recent results in the use of piezoelectric wafer active sensors (PWAS) in structural health monitoring applications. PWAS are small, inexpensive, non-invasive, elastic wave generators/receptors that can be easily affixed to a structure and interrogated on demand. PWAS are wideband non-resonant devices. They can be wired into sensor arrays and connected to data concentrators and wireless communicators. However, the development of PWAS technology is not yet complete, and a number of issues have still to be resolved. This paper has presented recent results obtained in using the PWAS for structural health monitoring. The new results presented were obtained in the following directions: (i) tuning the PWAS of on different plate thicknesses and of asymmetric PWAS geometries (rectangular plan forms) to excite and detect different Lamb wave modes in selective directions; (ii) PWAS durability and survivability under a number of exposures; (iii) steering beam large-area coverage (embedded ultrasonic structural radar - EUSR); and probability of detection (POD) with PWAS probes (an initial investigation).

Analytical methods and experimental results in the tuning of various Lamb wave modes with PWAS transducers have been presented. Frequencies up to $700 \mathrm{kHz}$ were investigated. Plate thicknesses of 1.05 $\mathrm{mm}$ and $3.15 \mathrm{~mm}$ were considered. Square, round, and rectangular PWAS were tested. It was found that the theoretical models are validated by the experimental results. The effect of plate thickness was also verified, and it was found that the use of PWAS on thicker plates results in more Lamb modes (A0, A1, S0) being simultaneously excited, as predicted by the theory. An effective PWAS dimension, which is slightly less that the physical dimension, had to be considered in order to obtain an exact match between theory and experiments. This aspect is attributed to the effect of stress diffusion in the adhesive layer that joins the PWAS to the structure. Regarding the use rectangular PWAS, it was found that directional tuning is achieved when the length and width dimensions are clearly different. The results can be successfully used in the design of active SHM systems to be installed on operational structures.

The durability and survivability of the PWAS transducers under various exposures (temperature cycling, freeze-thaw, outdoor environment, operational fluids, large strains, fatigue load cycling) was considered. The large strain tests indicated that the PWAS remain operational up to at least 3000 microstrain and fail beyond 6000 micro-strain. The fatigue cyclic loading, conducted up to millions of cycles, showed that the substrate material always failed before the PWAS transducers. These results gave us confidence in this new technology and opened the path toward installation on realistic aerospace specimens. For longer-term durability and survivability results, further tests are being planned for subsequent studies.

The EUSR steering beam method based on PWAS phased-arrays has been refined to increase it power of detection for various structural defects. It has been also proved that EUSR performance is not affected 
by structural curvature typical of aircraft installation. The initial investigation of PWAS POD has indicated that crack as small as $1.57 \mathrm{~mm}$ can be detected directly and imaged relatively easily.

Overall, the results presented in this paper confirm that PWAS transducers technology has a good potential for implementation in active SHM systems. Further research work should focus on refining the theoretical and experimental results and then testing the method on realistic aerospace structures towards full industrial implementation.

\section{ACKNOWLEDGMENTS}

The financial support of National Science Foundation award \# CMS 0408578, Dr. Shih Chi Liu, program director; Air Force Office of Scientific Research grant \# FA9550-04-0085, Capt. Clark Allred, $\mathrm{PhD}$, program manager; Air Force Research Laboratory through Universal Technologies, Inc. are gratefully acknowledged. The author is indebted to his former and present graduate students for their indirect contributions to this paper through the cited references.

\section{REFERENCES}

Bottai, G.; Giurgiutiu, V. (2005) "Simulation of the Lamb Wave Interaction between Piezoelectric Wafer Active Sensors and Host Structure”, SPIE's $12^{\text {th }}$ International Symposium on Smart Structures and Materials and $10^{\text {th }}$ International Symposium on NDE for Health Monitoring and Diagnostics, Sensors and Smart Structures Technologies for Civil, Mechanical, and Aerospace Systems Conference, San Diego, CA, 7-10 March, 2005, paper \# 5765-29

Doane, J.; Giurgiutiu, V. (2005) “An Initial Investigation of the Large Strain and Fatigue Loading Behavior of Piezoelectric Wafer Active Sensors”, SPIE's $12^{\text {th }}$ International Symposium on Smart Structures and Materials and $10^{\text {th }}$ International Symposium on NDE for Health Monitoring and Diagnostics, Sensors and Smart Structures Technologies for Civil, Mechanical, and Aerospace Systems Conference, 6-10 March 2005, San Diego, CA. paper \# 5765-130

Giurgiutiu, V. (2003) "Lamb Wave Generation with Piezoelectric Wafer Active Sensors for Structural Health Monitoring”, SPIE's $10^{\text {th }}$ Annual International Symposium on Smart Structures and Materials and $8^{\text {th }}$ Annual International Symposium on NDE for Health Monitoring and Diagnostics, 2-6 March 2003, San Diego, CA, paper \# 5056-17

Giurgiutiu, V.; Bao, J. (2002) "Embedded Ultrasonic Structural Radar for the Nondestructive Evaluation of ThinWall Structures” Proceedings of the 2002 ASME International Mechanical Engineering Congress, November 1722, 2002, New Orleans, LA, paper \# IMECE2002-39017

Giurgiutiu, V.; Yu, L.; Thomas, D. (2004) "Embedded Ultrasonic Structural Radar with Piezoelectric Wafer Active Sensors for Damage Detection in Cylindrical Shell Structures”, 45 ${ }^{\text {th }}$ AIAA/ASME/ASCE/AHS/ASC Structures, Structural Dynamics \& Materials Conference and $12^{\text {th }}$ AIAA/ASME/AHS Adaptive Structures Forum, Palm Springs, CA, 19-22 Apr. 2004, paper \# AIAA-2004-1983

Giurgiutiu, V.; Zagrai, A. N. (2002) “Embedded Self-Sensing Piezoelectric Active Sensors for Online Structural Identification”, ASME Journal of Vibration and Acoustics, Vol. 124, January 2002, pp. 116-125

Raghavan A., Cesnik C. E. S. (2004) "Modeling of piezoelectric-based Lamb-wave generation and sensing for structural health monitoring"; Proceedings of SPIE - Volume 5391 Smart Structures and Materials 2004: Sensors and Smart Structures Technologies for Civil, Mechanical, and Aerospace Systems, Shih-Chi Liu, Editor, July 2004, pp. 419-430

Yu, L.; Giurgiutiu, V. (2005) “Multi-damage Detection with Embedded Ultrasonic Structural Radar Algorithm using Piezoelectric Wafer Active Sensors through Advanced Signal Processing” NDE for Health Monitoring and Diagnostics, Health Monitoring and Smart NDE of Structural and Biological Systems Conference, San Diego, CA, 7-10 March, 2005, paper \# 5768-48 